\title{
Gestión clínica no deshumanizada
}

\section{Non-dehumanized clinical management}

\author{
Pablo Arango • Chía, Cundinamarca (Colombia)
}

"La vida no es un negocio, es un arte"

Proverbio chino

\section{Resumen}

La gestión clínica abarca muchos campos, es útil para mejorar la administración de una institución y para mejorar la atención médica, pero estos dos objetivos pueden entrar en conflicto. Se hacen unas breves consideraciones sobre lo que debe ser la gestión clínica desde el punto de vista de la humanización y de la bioética, así como el respeto hacia el ser humano mediante una buena práctica médica.

(Acta Med Colomb 2012; 37: 38-40)

Palabras clave: gestión clínica, humanización.

\begin{abstract}
Clinical management encompasses many fields. It is useful to improve the administration of an Institution and its medical care, but these two goals can come into conflict. Some brief remarks are made about how clinical management should be from the point of view of humanization and bioethics, such as respect towards patient and best practices towards him or her as an integral human being.
\end{abstract} (Acta Med Colomb 2012; 37: 38-40)

Key words: clinical management, humanization.
Dr. Pablo Arango Restrepo: PhD. Profesor Departamento de Bioética, Profesor Cirugía Ortopédica Facultad de Medicina Universidad de La Sabana, Campus Universitario Puente del Común, Chía, Cundinamarca (Colombia).

Correspondencia: Dr. Pablo Arango Restrepo. Chía, Cundinamarca (Colombia).

E-mail: pablo.arango@unisabana.edu.co, pabloarangor@gmail.com

Recibido: 13/X/2011 Aceptado: 30/I/2012
Todo es cada día más complejo, es evidente el afán de muchos por hacer las cosas bien, hay muchos sistemas de gestión, hay procesos que funcionan bien y otros no tanto que es necesario mejorar. En nuestro país, en el campo de la salud, se está trabajando en esto tanto a nivel privado con gubernamental (1) y los médicos cada día estamos más involucrados en los procesos de gestión.

Gestión clínica es un término moderno y muy amplio que incluye todos los aspectos de la administración (hoy llamados dirección y gestión) de una institución de salud que en términos generales están focalizados en dos grandes áreas de un hospital, un área es la parte administrativa que se preocupa por las políticas laborales, sistemas de contratación del personal, manejo financiero, aspectos locativos y de accesibilidad, mercadeo, manejo de la información administrativa y otra gran área responsable de la parte médica, la información médica (2) como es la historia clínica, la atención de los pacientes (atención primaria y especializada) y dentro de la atención médica se incluyen una serie de protocolos de manejo que buscan hacer más efectivos y eficaces los esfuerzos por brindar salud de buena calidad, satisfacer al paciente (3), y evitar el error médico. Estas dos áreas, en la práctica, tienen mucha interrelación y es necesario que la tengan para el buen funcionamiento de la institución que redunda en beneficio de los pacientes.

Una de las razones de estos esfuerzos es querer controlar el gasto de salud que en todos los países es muy alto por los continuos avances en salud y la aparición de enfermedades de alto costo, muchas de las cuales marchan de la mano con el envejecimiento de la población, que han disparado los costos y no hay sistema que los resista. Al lado de querer controlar el gasto está también el deseo de dar una atención de calidad que por lo general exige un aumento del gasto.

Los administradores de la salud se ven con frecuencia ante el dilema de controlar el gasto o mejorar la calidad. La idea general con la aplicación de modelos de gestión clínica es que si logramos identificar e implantar procesos eficaces lograremos bajar los costos y disminuir los errores.

Un aspecto importante de la calidad es el evitar el error médico, que es causante muchas veces de la muerte de los pacientes o de lesiones graves, este es un punto que cada día cobra más importancia y en el que se están logrando resultados positivos.

Hay dos definiciones importantes que debemos tener presentes: 
Gestión son las acciones coordinadas orientadas al logro de los objetivos institucionales (eficacia) con el menor uso de recursos posible (eficiencia) (4).

La gestión clínica en concreto busca incrementar la eficiencia y la calidad de las prestaciones sanitarias otorgadas por las unidades asistenciales.

Es la gestión de los procesos clínicos, de todas las actividades que en ellos se engloban y los recursos necesarios para llevarlos a cabo.

El John Hopkins Hospital (USA) señala la importancia de la participación del médico en la gestión cuando dice que es el proceso de rediseño organizativo cuyo objetivo es involucrar al profesional sanitario, esencialmente médico, en la gestión de los recursos utilizados en su propia práctica clínica.

Indudablemente el médico debe estar involucrado en el proceso, es de alguna manera el actor principal, y la medicina es una ciencia y un arte, en cuanto a ciencia se basa en una serie de conocimientos científicos hasta cierto punto universales y por otro lado es un arte, reconoce que cada paciente es diferente, que hay situaciones que no se pueden generalizar y por tanto los tratamientos se deben individualizar. En este punto converge también el criterio médico y por tanto la autonomía del médico para señalar el mejor tratamiento para su paciente, en particular teniendo en cuenta no sólo un dato clínico concreto, como puede ser la alergia a un medicamento o la no sensibilidad de un germen a un determinado antibiótico, sino el paciente como ser persona, como ciudadano de una determinada clase social $\mathrm{y}$ con un determinado apoyo familiar y social.

La gestión clínica se apoya en el campo médico en las Guías de Práctica Clínica, que son útiles porque estandarizan la práctica médica al sintetizar la información científica, ofrecen la mejor atención médica basada en la evidencia científica ayudando en la toma de decisiones, mejoran la utilización de los recursos y contienen los gastos (5), pero que no son palabra de Dios por lo que señalábamos antes, cada paciente es diferente en muchos aspectos.

La consistencia es un término moderno para indicar que la empresa debe cumplir con la naturaleza de su negocio, que en este caso de las empresas de salud es sanar personas. Las empresas de salud tienen una misión muy clara, brindar salud, no quiere decir que se desentiendan por completo de la consecución de recursos económicos, de economizar, pero debe haber una escala de valores perfectamente definida y respetada.

El afán de realizar una gestión clínica buena puede llevar a los administrativos a imponer por ejemplos políticas de empleo o salariales injustas, exigir jornadas de trabajo inadecuadas, reducir tiempos de consulta o disminuir tiempos de hospitalización cuando esto no es lo más conveniente desde el punto de vista médico. A un caso de esto me voy a referir y a partir de él hacer algunos comentarios éticos.

Conocí por referencia un caso de una institución que dentro de su plan de gestión clínica había reducido el tiempo que transcurría desde que un(a) paciente mayor de 65 años, del régimen subsidiado, con fractura de cadera, consultaba al servicio de urgencias y salía una vez intervenido para su casa: el tiempo se había reducido de 8 o 9 días a 4 o 5 . ¿Cómo lo hicieron? Cuando consultaba a urgencias se tomaba la radiografía inmediatamente, al hacerse el diagnóstico se solicitaban todos los exámenes de laboratorio, la valoración cardiológica y de anestesia, se informaba al cirujano, se intervenía en menos de 48 horas y dos días después era referido a su casa. Los pacientes eran del régimen subsidiado, por tanto Sisben I y II.

Los hospitales han existido hace muchos años, en su momento fueron un avance importante en la medicina, se pudo prestar una atención más esmerada y cuidada a los enfermos. Hace 35 años pacientes con patologías pequeñas como una hernia inguinal se hospitalizaba la víspera, el anestesiólogo los visitaba y premedicaba, algo que hoy en día se echa de menos. A estos pacientes se les reservaba sangre, se intervenían al día siguiente de la hernia, y si todo iba bien, se enviaba a la casa un día después. Esto evolucionó y llegó la modalidad de la cirugía ambulatoria, se vio que no todo paciente necesitaba estar hospitalizado. Al principio se fue muy prudente, se hicieron cursos sobre las condiciones para llevar al paciente a un programa de cirugía ambulatoria, y esto ha funcionado bastante bien, excepto por el hecho de que así como antes se abusaba de las hospitalizaciones ahora se abusa de la cirugía ambulatoria y de la corta estancia. Los ortopedistas al principio, hacíamos en forma ambulatoria cirugías pequeñas, por ejemplo cirugías de mano, ahora se hacen osteosíntesis de antebrazo, o de pierna, reconstrucciones del ligamento cruzado también, y estos pacientes son los que están llamando a su médico para quejarse de dolor, de molestias, como que no sienten el pie, que el vendaje está apretado, que no pueden mover los dedos, y muchas veces pensamos para nuestros adentros "ese paciente debería estar en la clínica".

¿Por qué se ha llegado a esto? Yo diría que hay dos razones principales, la primera la presión de los administrativos y de los aseguradores para que el paciente no esté hospitalizado, y la segunda, porque al médico no le queda tiempo para ir a evolucionar o dar salida, y con el paciente en la casa "se quita este problema de encima".

Hay otro grupo de pacientes, que ya de antemano se sabe que no son ambulatorios, pero que de alguna manera se ven envueltos en esta corriente o tendencia a disminuir hospitalización. Es el caso de pacientes con fracturas de cadera, mayores de 65 años, que son recibidos en la institución, intervenidos y dados de alta a las 48-72 horas.

Se aducen razones diversas como que lo importante ya se hizo, los hospitales son lugares peligrosos por la posibilidad de adquirir infecciones nosocomiales, en su casa va a estar mejor, etc. En esto debemos ser analíticos: evidentemente lo dicho puede ser cierto en algunos casos, pero en otros con seguridad que no. Si el paciente es de un estrato social alto, con una familia en la que no todos trabajan, se puede enviar a la casa y allí con seguridad habrá quien le suminis- 
tre la medicación que incluye los analgésicos, la profilaxis antiembolica usualmente parenteral, se podrá solicitar una fisioterapeuta para la casa, y habrá alguien que le ayude a sentarse en una silla, hacer sus necesidades fisiológicas. Pero resulta que los pacientes de un estrato social alto en nuestro país son el 2-3\%. Casi el 65\% de la población son Sisben I, II, y III, son pacientes que no tienen quien les ayude, en su casa no hay condiciones higiénicas mínimas, si en la clínica el riesgo es una infección nosocomial aquí es una infección adquirida en comunidad, ¿quién le va a aplicar la profilaxis antiembolicas (en el supuesto de que se la hubieran suministrado porque comprarla no es posible)?, ¿qué tipo de terapias le van a hacer?, ¿quién le va ayudar en su casa a sus necesidades fisiológicas?, ¿qué tipo de cuidado va a recibir cuando en esa casa no hay lavamanos, el piso es de tierra y está llena de animales domésticos y no domésticos? La respuesta a estas preguntas posiblemente es que nadie. ¿Qué hacer? Posiblemente hay muchas respuestas: que haya más equidad, que no haya clases sociales, crear programas de apoyo médico domiciliario, etc. Todo esto es verdad, ¿pero por qué no recordamos que precisamente para este tipo de casos se crearon los hospitales e instituciones de salud? ¿Qué afán tenemos de mandar al paciente a la casa cuando donde mejor va a estar es precisamente en el hospital? Seguramente a los economistas esta solución no les va a gustar, pero es que es necesaria. Los economistas han ayudado a mejorar algunos aspectos de la salud, pero tienen un gravísimo problema y es que no son médicos, mucho -no todos- no tienen sensibilidad en el corazón para comprender el dolor ajeno, todo lo observan por el prisma del dinero y resulta que en la vida hay muchísimos otros aspectos por considerar. El ganar dinero es un fin lícito, pero no puede ser el único fin que se busque, las empresas tienen lo que se conoce hoy en día como responsabilidad social empresarial y no se puede olvidar el factor humano, que es un activo importantísimo en la sociedad.

¿Qué decir de compañías que en recesión lo que hacen es despedir gente cuando tienen en sus arcas ganancias de 25 años? En nuestro país los bancos ganan billones cada año. Son tan pobres que lo único que tienen es dinero.

Humanización de la salud es el tema. Gestión clínica eficiente sí, pero con alma. Los médicos debemos participar en la gestión clínica de las instituciones, somos los más indicados para hacer ver la deshumanización porque supuestamente somos los que hablamos con nuestros pacientes y los conocemos a ellos y sus circunstancias y no podemos permitir que se cometan atropellos.
La administración es una ciencia humana, las facultades de administración debieran estar en el grupo de facultades humanísticas de las universidades pero desafortunadamente están más cerca de las facultades técnicas y la técnica nos ha dado enormes adelantos y ha permitido avances significativos en el campo de la salud, pero la técnica no puede dar razón de quién es el hombre, de quién es el ser humano. La técnica se mueve en un campo muy práctico, de lo físico, de lo que tiene valor monetario.

¿Cuánto vale un ser humano? Los seres humanos no tenemos precio; filósofos como Kant ya nos explicaron hace años que los seres humanos no tienen precio, lo que tiene precio se puede comprar, los seres humanos tenemos dignidad, algo que nos pone muy por encima de las cosas. La dignidad es de todos o no es de nadie, la dignidad se nos da, es igual para todos.

Dice el filósofo español Leonardo Polo se suele afirmar que los negocios son los negocios; y hay que responder: los negocios no son los negocios, sino que los negocios son negocios si son éticos (6), y dice Juan Pablo II: la economía no puede imponer los modelos y el ritmo del desarrollo, y aunque es justo proveer a las necesidades materiales, nunca se han de ahogar los valores del espíritu. Lo verdadero debe prevalecer sobre lo útil, el bien sobre el bienestar, la libertad sobre las modas y la persona sobre la estructura (7).

Ya decía anteriormente que ganar dinero es algo lícito, pero desde luego hay que hacerlo de manera adecuada, honesta, es decir, con respeto, sin explotar a los demás, sin usura, sin engaño, de una manera proporcionada para que no sea explotación. ¿Cuál debe ser la principal ganancia de las empresas de salud? La salud de su gente. No sólo ganar dinero. Servir. Dignificar la vida de las personas. Hacerla más humana. Esta es la consistencia de la empresa que se espera, es decir que sea consecuente con el objeto humano para lo que fue constituida.

\section{Referencias}

1. www.minproteccionsocial.gov.co/.../DocNewsNo15384DocumentNo2568.pdf 3 de julio de 2009

2. Abizanda CR, Ferrándiz SA, Reig VR. PDMS o UDMS. Dos perspectivas de gestión clínica en Medicina Intensiva. Med Intensiva 2008; 32: 7.

3. Ruiz LP, Alcalde EJ, Rodríguez-Cuellar E y col. Proyecto nacional para la gestión clínica de procesos asistenciales. Tratamiento quirúrgico del cáncer colorrectal. I. Aspectos generales. Cir Esp 2002; 71: 173-80

4. Alberto Galli, en www.mutualadim.org.ar/Ponencias/2010CI/Herramientas _de.../ Galli.ppt, consultada 1 de junio de 2011.

5. www.higiene.edu.uy/cefa/uti5/IntGestClin.ppt 3 de julio de 2009.

6. Polo, Leonardo. Ética, 1995, Eunsa, p. 19

7. Juan Pablo II. Discurso, 9 de abril de 2001. 